\title{
Diagnostic Difficulties in a Severely Ill HIV Patient with Multiple Superinfections - A Case Report
}

\author{
Cristina Mănășturean ${ }^{1}$, Cristiana Oprea22, Dan Oțelea ${ }^{3}$, Anca Meda Georgescu1,4 \\ 1 Infectious Diseases Clinic I, Mures County Hospital , Târgu Mureș, Romania \\ 2 Victor Babeș Clinical Hospital of Infectious and Tropical Diseases, Bucharest, Romania \\ 3 Molecular Diagnostics Laboratory, National Institute for Infectious Diseases, Bucharest, Romania \\ 4 University of Medicine, Pharmacy, Science and Technology of Târgu-Mureș, Romania
}

\begin{abstract}
Introduction: As chronic HIV infection is prone to co-infections more than any other infectious condition, many severely immune-depressed patients require advanced diagnostic investigations and complex treatment.

Case report: The case of a 30-year-old severely immune-depressed patient with AIDS, who developed neurological impairment and was diagnosed with encephalitis is presented. Multiple diagnostic approaches had to be used in order to identify the etiologic agents responsible for the clinical, immunological and biological evolution. Despite using advanced laboratory investigations and complex treatment, the patient developed multiple organ dysfunction syndromes that led to a fatal outcome.
\end{abstract}

Conclusions: Establishing etiologic relations and treatment priorities in patients with severe immunodeficiency and co-infections can prove difficult, underlining the need of rapid syndromic testing.

Keywords: HIV, immunodeficiency, superinfections, encephalitis

Received: 9 August 2019 / Accepted: 31 August 2019

\section{INTRODUCTION}

Chronic HIV infection is prone to co-infections more than any other infectious condition as the HIV virus cripples the immune system and allows reactivation of dormant pathogens or increases susceptibility to exogenous pathogens [1]. These co-infections further fuel the immune activation that characterizes HIV infection even after initiation of antiretroviral therapy. Morbidity and mortality by opportunistic diseases have recently decreased but infections with Cytomegalovirus (CMV) and Papillomavirus (HPV), tuberculosis, hepatitis $\mathrm{C}$ and $\mathrm{B}$ are still major health issues for people living with HIV (PLWH). Quite frequently the severely immunedepressed patients end up with several infections and organ failures in an intensive care unit.

Progressive multifocal leukoencephalopathy (PML) is a demyelinating disease first reported as a complication of chronic lymphocytic leukaemia and Hodgkin's disease. It is caused by JCV, a polyomavirus, and had a rapidly increasing incidence in the mid-1980s associated with the HIV/AIDS epidemic. The introduction of effective combination antiretroviral therapy (cART) led to a large fall in the number of cases of PML and markedly improved survival rates. However, patients with a $\mathrm{HIV}$-associated disease still account for roughly $80 \%$ of all reported cases of PML, and over half of them die within two years of diagnosis [2].

\section{CASe presentation}

A thirty-year-old male patient with AIDS was admitted to the Infectious Diseases Clinic 1, Târgu-Mureș, Romania in January 2019 because of left temporo-parietal headache, left side face paraesthesia, hearing impairment, vertigo and abnormal gait. The patient belonged to the Romanian HIV cohort of young adults who have been nosocomially infected very early in childhood [3]. The diagnosis was made in 2003. His medical history included pulmonary tuberculosis treated in 2005. The antiretroviral therapy consisted of emtricitabine $200 \mathrm{mg}$ qd, tenofovir disoproxil fumarate $245 \mathrm{mg}$ qd and raltegravir $800 \mathrm{mg}$ qd. He had not adhered to the 
recommended drug regimen and had intermittently self-administered the treatment during the preceding six months.

At the time of admission his physical examination was dominated by neurologic deficits: facial asymmetry, horizontal left-sided nystagmus, wide-based gait and a positive Romberg test. The patient had a CD4 count of 10 cells $/ \mathrm{mL}$. Ophthalmological examination showed lagophthalmos, proptosis and abduction deficit of the left eye and ophthalmoscopy revealed hyperpigmented macular scars in the right eye and brush-fire microhaemorrhages and retinal exudative oedema in the left eye, interpreted as active chorioretinitis lesions compatible with CMV infection.

A lumbar puncture revealed clear cerebrospinal fluid (CSF), with $7 / 3$ lymphocytes/dL, a glucose level of $63.17 \mathrm{mg} / \mathrm{dL}$ and a protein level of $67.6 \mathrm{mg} / \mathrm{dL} .500$ mg intravenous acyclovir three times a day was initiated for a suspected herpes virus infection. Mannitol was administered in association with dexamethasone corticotherapy.

Tongue scrapings were positive for Candida albicans and Candida krusei was found in the urine and therefore fluconazole $150 \mathrm{mg}$ qd was administered orally.

A brain magnetic resonance imaging (MRI) scan revealed findings suggestive of vascular lesion sequelae. The patient's condition deteriorated in spite of treatment, and further impaired vision and hearing, muscle hypotonia, crural paresis, ataxic gait worsened, the later making walking impossible. He also developed intercostal herpes zoster and a productive cough. PML was suspected. After six weeks of treatment in our clinic, in order to confirm this diagnosis he was transferred to Dr. Victor Babeș Clinical Hospital of Infectious and Tropical Diseases, (Bucharest, Romania).

On admission, to this hospital, a polymerase chain reaction test (PCR) detected Cytomegalovirus (CMV) and Varicella Zoster virus (VZV) DNA in the cerebrospinal fluid. Testing for the JC virus was negative.

The patient was treated with intravenous ganciclovir $250 \mathrm{mg}$ twice a day followed by $450 \mathrm{mg}$ oral valgancyclovir thice a day.

The patient's CD4 cell count was 12, with a HIV plasma viral load of 117.050 copies/mL and a CSF viral load of 2180 copies $/ \mathrm{mL}$. Antiretroviral drug resistance genotypic testing showed no resistance mutations. A chest radiograph showed an ill-defined round opacity measuring $20 / 30 \mathrm{~mm}$ situated centrally in the right lung and the Lowenstein-Jensen culture from his sputum was positive for Mycobacterium tuberculosis. Antituberculous therapy was initiated with $250 \mathrm{mg}$ oral isoniazid once daily, $450 \mathrm{mg}$ oral rifampicin once daily, $1 \mathrm{~g}$ oral pyrazinamide once daily and $800 \mathrm{mg}$ oral ethambutol once daily.

He developed cognitive impairment with psychomotor agitation, insomnia, hallucinations and heteroaggressiveness and therefore psychiatric medication with diazepam, tiapride and tradozone was administered. Urinary catheterization was required for bladder globus.

A second brain MRI was performed revealing a significant, bilateral, circumferential thickening of the of dura mater most likely of an inflammatory nature. There was also a slight enlargement of the subdural space, and a thin lamellar lesion in the right subdural space, with high T1, average T2 signal and water restriction of uncertain cause.

Another finding was a small lesion with ring-like contrast intake in the left lenticulocapsular region with minimal increase in size, possibly due to toxoplasmosis, but which was ruled out by a negative test for Toxoplasma IgG antibodies.

A diagnosis was made of encephalitis with CMV and VZV, pulmonary tuberculosis, intercostal herpes zoster, CMV chorioretinitis, oral candidiasis, urinary tract infection with Candida krusei occurring in a stage C3 HIV positive patient.

Following another six weeks and on completing the antiviral treatment, his condition slightly improved. He was transferred to the Infectious Diseases Clinic, Sibiu, Romania

While in hospital he developed Clostridium difficile colitis, which was treated with vancomycin and metronidazole.

The patient also developed acute hepatic failure, recurrent haematochezia and prolonged p t (INR 3.25) that required administration of antihaemorrhagic agents and blood transfusion.

The patient was discharged after five weeks of hospitalization and six months after the first admission.

He was readmitted two months later to the Infectious Diseases Clinic, Sibiu with a recurrence. He was underweight, with a body mass index of $17.57 \mathrm{~kg} / \mathrm{m}^{2}$ and presented with fever and jaundice.

He did not respond to treatment with blood transfusions and granulocyte-colony stimulating factor. 
Over the next two weeks the patient developed multiple system organ failures leading to death.

\section{DISCUSSION}

This case reflects the fact that in HIV infected patients co-infections can rapidly evolve to life threatening conditions requiring intensive care support. Twenty five percent of the 1.5 million yearly deaths of HIV infected patients are attributed to tuberculosis [4], about 25\% are co-infected with $\mathrm{HCV}$, and near $10 \%$ with HBV [5]. The patient in this case was first diagnosed as being HIV positive in 2003, and was first treated for pulmonary tuberculosis in 2005 and currently after relapse. At the time of hospital admission he was profoundly immunosuppressed with a CD4 count of 10 cells $/ \mu \mathrm{L}$. PLWH with lower CD4 counts more likely develop TB compared with those with better immune status at HIV diagnosis [6], and CMV still contributes to morbidity and mortality in patients who have low $\mathrm{CD} 4{ }^{+}$T-cell counts [7]. Reactivation of CMV in immunocompromised hosts can cause life-threatening complications [7]. 85\% of all CMV pathology in HIV-infected subjects with advanced disease is CMV retinitis, leading to blindness in many cases. Ophthalmoscopy showed active chorioretinitis lesions, macular scars. CMV and VZV DNAs were detected in CSF by PCR.

Despite receiving treatment, the patient's condition deteriorated, with severe neurological signs, vision and hearing impairment, muscle hypotonia, crural paresis and worsened ataxic gait. He also developed intercostal herpes zoster.

In CMV infections, as CNS imaging findings are nonspecific, diagnosis is made by identifying CMV in CSF through PCR testing [8]. Blood assays to detect CMV DNA or antigen are not recommended for diagnosis of CMV end-organ disease[9].

CMV neurologic disease is diagnosed on the basis of a compatible clinical syndrome and PCR [10]. Symptoms typically include altered mental status, delirium, confusion, weakness, or urinary retention, all observed in the present case. CSF levels of IgM antibodies for CMV virus were undetectable, supporting the suspicion that this was a reactivation although the lack of response might also be the effect of immune-depression. When the patient's neurological status deteriorated, it was suspected that he may had developed PML, as its classic clinical presentation includes subacute to chronic focal neurological deficits. The most common clinical manifestations are weakness (80\%), disturbances of visual field (50\%) and cognitive deterioration. [11]. JCV DNA was not detected in the patient's CSF but the amount of virus in the CSF can sometimes fall below detection thresholds. Therefore, it should be noted that a negative JCV PCR result does not rule out PML. The overall neurological disturbances as well as MRI changes in the patient can be accounted for by any of the coexisting conditions. CMV reactivation in immunocompromised patients manifests as acute ventriculitis. T2 hyper intensities and contrast enhancement were present along the wall of the ventricles. The typical presentation of VZV encephalitis is a vasculopathy causing ischemic infarction and arterial stenosis. Typical MRI findings of PML are white matter lesions in different brain areas, predominantly the parieto-occipital lobes. The patient usually shows little or no inflammation with hyper intense in T2-weighted and FLAIR sequences and hypo intense in T1-weighted scans in relation to the white matter involvement, with no contrast enhancement and no mass effect on MRI. The patient's brain MRI showed modifications which may represent vascular lesion sequalae.

None of the viruses he was infected with could be entirely ruled out as the causing agent for brain damage but CMV was considered the most likely, due to positive PCR associated with ophthalmic lesions.

Given the poor outcomes in many patients with CMV-related neurologic disease, some experts recommend initiation of both IV ganciclovir and IV foscarnet, but there are significant toxicities associated with these medications among which are anaemia, neutropenia, thrombocytopenia, nausea, diarrhoea and renal dysfunction. These complications were also present in this case, although medication with foscarnet was not administered, and the multiple organ dysfunctions were considered as due to the prescribed medication consisting of fluconazole, izoniazid, rifampicin, pyrazinamide, ethambutol.

Resistance to antiretroviral drugs was tested, but the fact that the patient intermittently interrupted his treatment for six months must be taken into account when assessing the HIV-1 virologic failure and the lack of mutations. The lack of virologic and immunologic response, as well as the continuous multiplication of wild type virus during treatment suggest that the biodisponibility of cART might have been suboptimal. Therapeutic drug monitoring was not available at the time. 
Available online at: www.jccm.ro

Prior to cART the median survival with neurologic CMV was 4.6 weeks from the onset of symptoms and in hospital mortality $38 \%$, though there is no data on CMV encephalitis outcomes after the introduction of cART [7].

The patient's evolution comprised multiple hospitalizations, interdisciplinary approach, complex therapy and, despite all that, a fatal outcome six months after the initial admission.

\section{CONCLUSIONS}

Managing patients with severe immunodeficiency and several co-infections can prove dauntingly challenging. Establishing etiologic relations and treatment priorities can be difficult, not least in patients with precarious biologic conditions. The increasing availability of rapid syndromic testing might help shorten the diagnostic process and improve the success rate of prescribed treatment modalities.

\section{ACKNOWLEDGMENTS}

Part of the data were communicated as oral presentation at the National Conference of Infectious Diseases 8-10 May 2019, Timișoara, Romania.

\section{CONFLICT OF INTEREST}

None to declare

\section{BIBLIOGRAPHY}

1. Zanoni BC, Gandhi RT. Update on opportunistic infections in the era of effective antiretroviral therapy. Infect Dis Clin North Am. 2014;28:501-18.
The Journal of Critical Care Medicine 2019;5(4) • 139

2. Hirsch HH, Kardas P, Kranz D et al. The human JC polyomavirus (JCPyV): virological background and clinical implications. APMIS. 2013;121:685-727.

3. Hersh BS, Popovici F, Jezek Z, et al. Risk factors for HIV infection among abandoned Romanian children. AIDS. 1993;7(12):161724.

4. World Health Organization. Global Tuberculosis Report. 20th ed. 2015. Available at: https://apps.who.int/iris/ handle/10665/191102

5. Centers for Disease Control and Prevention. Monitoring selected national HIV prevention and care objectives by using HIV surveillance data-United States and 6 dependent areas, 2017. HIV Surveillance Supplemental Report 2019;24(No. 3). Available at: http://www.cdc.gov/hiv/library/reports/ hivsurveillance.html. Published June 2019

6. Sterling TR, Pham PA, Chaisson RE. HIV infection-related tuberculosis: clinical manifestations and treatment.Clin Infect Dis. 2010; 50(Suppl 3): S223-S230.

7. Springer KL, Weinberg A. Cytomegalovirus infection in the era of HAART:fewer reactivations and more immunity. J Antimicrob Chemother. 2004;54:582-86.

8. Stern-Ginossar N, Weisburd B, Michalski A, et al. Decoding human cytomegalovirus. Science. 2012;338:1088-93.

9. CDC. Guidelines for the prevention and treatment of opportunistic infections in HIV-infected adults and adolescents: recommendations from the Centers for Disease Control and Prevention, the National Institutes of Health, and the HIV Medicine Association of the Infectious Diseases Society of America. October 2015.

10. Newcomb G, Mariuz P, Lachant D. CMV Encephalitis/Radiculitis: the difficulty in diagnosing in an intubated patient. Case Reports in Critical Care. 2019;ID 8067648.

11. Cinque P, Koralnik IJ, Gerevini S, et al. Progressive multifocal leukoencephalopathy in HIV-1 infection. Lancet. 2009;9:62536. 